\title{
Multifunctional contamination-resistant coatings
}

\author{
Nadja Felde ${ }^{1, *}$, Anne Gärtner ${ }^{1}$, Stefan Schwinde ${ }^{1}$ and Sven Schröder ${ }^{1}$ \\ ${ }^{1}$ Fraunhofer Institute for Applied Optics and Precision Engineering, Albert-Einstein-Strasse 7, 07745 Jena, Germany
}

\begin{abstract}
Surface contaminations can critically affect the performance of optical surfaces, in particular with respect to light scattering, optical losses, and laser stability. Thus, avoiding contaminations and reducing contamination-induced effects is of particular interest in the manufacturing of optical systems. By combining a specific thin film design with a tailored structural design, contamination-resistant coatings with a high optical quality can be realized. Most important is the balance of self-cleaning- and light scattering-relevant surface roughness components.
\end{abstract}

Surface contaminations of optical components can critically affect the performance of the entire optical system. Typical contaminations, which are most relevant in optical applications, can be for example dust particles from the environment or fluids from the manufacturing process. However, over the last years also biocontaminations have become increasingly critical.

To avoid contamination-induced effects, such as increased optical losses or reduced laser stability, optical coatings have to be applied, which reduce the accumulation of contaminations as well as their optical effects while keeping the optical quality at a high level.

Addressing this issue, a multifunctional coating was developed, which is characterized by the following functionalities (Fig. 1):

- Self-cleaning/contamination-repellent properties by wetting-relevant structuring

- Contamination-insensitivity by tailored optical coating design

- Broadband antireflective characteristics by structures with graded refractive index

- Low scattering by shifting the function-relevant structures to lateral scales, which are less relevant for light scattering.

For the self-cleaning/contamination repellent behaviour, the minimization of the adhesion forces between the surface and the contaminating particle or fluid is of particular importance. This is realized by tailoring the wetting properties of the coating to hydrophobic and oleophobic, severely water- and oil-repelling, properties. For this, a defined structuring and chemical functionalization [1] of the coating surface is needed. However, although required for the functionality, the increased surface roughness may have also a negative impact on the optical performance. This is the case as surface roughness leads to increased light scattering [2,3].

To address this problem, topographic, functional, and light scattering characterization methods have to be combined to individually analyse the specific properties and to comprehensively clarify their correlations to each other. This information is needed to selectively define the wetting- and light scattering-relevant spatial frequency range, which is based on power spectral density functions. This allows appropriate surface roughness structures of the multifunctional coating to be designed, which includes the shifting of the wetting-relevant surface structures to lateral scales that reduce or even prevent scatter losses $[1,4]$.

Nonetheless, complete avoiding of contaminations is inevitable over time. Thus, the multifunctional coating can further be equipped by an additional specific optical design. Within this design, adopting the layer thickness, the standing electric field strength at the position of the contaminating particle or fluid is modified $[6,7]$. Thus, contamination-induced light scattering properties can effectively be tailored.

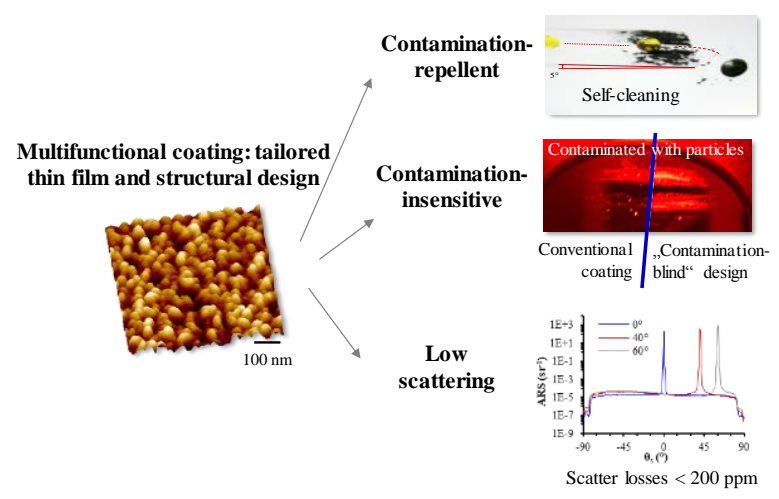

Fig. 1. Multifunctional contamination-resistant coating.

\footnotetext{
* Corresponding author: nadja.felde@iof.fraunhofer.de
} 
The resulting multifunctional coating can significantly enhance the wetting properties of a smooth optical surface. Compared to a smooth surface with functionalization, reaching only advancing and receding contact angles of $110^{\circ}$ and $95^{\circ}$, the multifunctional coating exhibits advancing and receding water contact angles of $155^{\circ}$ and $145^{\circ}$. Thus, water drops roll off at a tilt angle of the surface of $3^{\circ}$, ensuring particle contaminations to be removed [1].

Such repellence holds also true for contaminations with oil-containing liquids [8,9] such as human fingerprints and can even be transferred to reduce contaminations with molten metal debris [10]. However, with respect to biocontaminations both states, bacteria-adhesion and bacteria-colonization, have been shown to be impacted.

The main point, however, is that the optical quality of the components can be preserved after coating. In case of the multifunctional coating designed for applications in the visible spectral range the scatter losses are as low as $90 \mathrm{ppm}$ in the reflectance direction and results in less than $75 \mathrm{ppm}$ in the transmittance direction. Due to the function-relevant structuring additional broadband antireflective properties can be achieved.

\section{References}

1. N. Felde, L. Coriand, S. Schröder, A. Duparré, A. Tünnermann, Adv. Opt. Technol. 6, 387-394 (2017)

2. S. Schröder, M. Trost, M. Garrick, A. Duparré, X. Cheng, J. Zhang, and Z. Wang, Thin Solid Films 592, 248-255 (2015)

3. J. C. Stover, Optical Scattering: Measurement and Analysis (SPIE Press, Bellingham, Wash., 2012)

4. N. Felde, L. Coriand, A. Duparré, A. Tünnermann, J. Coat. Sci. Technol. 3, 100-108 (2016)

5. Patent application DE102014112133 A1, 2014

6. A. von Finck, S. Wilbrandt, O. Stenzel, S. Schröder, Adv. Opt. Technol. 6, 371-378 (2017)

7. Patent application DE 102016103339 A1, 2016

8. T. Oberbillig, N. Felde, M. Franz, L. Coriand, N. Dahms, K. Munderloh, K. Schultz, M. Fliedner, A. Duparré, Opt. Photonik 13, 14-18, (2018)

9. L. Coriand, N. Felde, and A. Duparré, "Wettability Behavior of Oleophilic and Oleophobic Nanorough Surfaces in Air or Immersed in Water, " in Advances in Contact Angle, Wettability and Adhesion, (John Wiley \& Sons, Inc., Hoboken, New York, 2018)

10. Patent application DE102018110251, 2018. 